$\underline{\text { Research Article }}$

\title{
Achieving and sustaining impact at scale for a newborn intervention in Nepal: a mixed-methods study
}

\author{
Stephen Hodgins ${ }^{1}$, Leela Khanal ${ }^{2}$, Nira Joshi ${ }^{3}$, Suzanne Penfold ${ }^{4}$, Sabita Tuladhar ${ }^{5}$, Parsu Ram Shrestha ${ }^{6}$, \\ Bikash Lamichhane ${ }^{6}$, Penny Dawson ${ }^{2}$, Tanya Guenther ${ }^{7}$, Samikshya Singh ${ }^{2}$, Ganesh Sharma ${ }^{8}$, Peter Oyloe \\ ${ }^{1}$ School of Public Health, University of Alberta, Edmonton, Alberta, Canada, ${ }^{2}$ John Snow Inc, Kathmandu, Nepal, ${ }^{3}$ New ERA, Kathmandu, Nepal, 4 \\ Independent consultant, Trnava, Slovakia, ${ }^{5}$ USAID Nepal, Kathmandu, Nepal, ${ }^{6}$ Ministry of Health, Government of Nepal, Kathmandu, Nepal, ${ }^{7}$ Saving \\ Newborn Live, Save the Children, Washington, DC, USA, 8 MEL project, CAMRIS Nepal, Kathmandu, Nepal, ${ }^{9}$ Independent consultant, Kathmandu \\ Nepal \\ Keywords: global health \\ https://doi.org/10.29392/joghr.3.e2019046
}

\section{Journal of Global Health Reports}

Vol. 3, 2019

\begin{abstract}
Background
Ten years ago - in the late 2000s - the government of Nepal was confronted with high infection-attributable neonatal mortality. There was new, locally-generated evidence that use of the antiseptic chlorhexidine for care of the newborn cord stump could substantially reduce the risk of such deaths but - at the time - no global-level recommendation. This paper traces the evolution of chlorhexidine introduction and scale up and documents program performance once the program had reached national scale, in terms of "implementation strength” and population-level "effective coverage."
\end{abstract}

\section{Methods}

Mixed methods implementation research, including a narrative account of the evolution of the program and results from a large, nationally-representative household survey of 3,661 live births over the preceding 12 months to assess use of chlorhexidine for newborn cord-care. These results are triangulated with survey findings from: a national survey of female community health volunteers (2015), a national survey of health facilities (2015), the Nepal Demographic and Health Survey (2016), and data from the government's Health Management Information System (2016-7).

\section{Findings}

The Ministry of Health, with USAID-funded technical assistance, first piloted the intervention in 4 districts beginning in 2009, decided in 2011 to move forward with national scale-up, and fully expanded delivery of the intervention to national scale by 2017. As of 2017 , chlorhexidine was applied to the cord of about $90 \%$ of newborns born in health facilities and about $40 \%$ of those born at home, for an overall population coverage of approximately $70 \%$. Chlorhexidine for cord-care was found to be in stock in $85 \%$ of public hospitals and $75 \%$ of peripheral level government health facilities offering childbirth care.

\section{Conclusions}

Important lessons can be drawn from this example on factors contributing to successful scale-up. However, the unreached 30\% remain particularly vulnerable, as they are disproportionately from among the less affluent; tailored strategies are needed to better reach them. Furthermore, circumstances on the ground are changing and it will require renewed efforts to sustain the gains that have been made.

In low-income countries, the burden of infection-attributable newborn deaths is approximately 100 -fold greater than in high-income countries. ${ }^{1}$ There are several factors accounting for this, notably differences in: resilience (birth weight and other factors), pathogen exposure (hygiene conditions and practices), availability of treatment, and use of preventive interventions. Among clinical preventive interventions addressing life-threatening infections in new- borns, antenatal tetanus immunization has been a major global health success story. In 1990, tetanus was 1 of the 4 leading causes of newborn death; at that time, WHO estimated it accounted for 787,000 newborn deaths per year. ${ }^{2}$ But by 2015 , tetanus was responsible for only $1 \%$ of newborn deaths, about 35,000 deaths/ year. ${ }^{3}$ Even with this momentous decline, neonatal mortality remains high in lowincome countries - above 20 deaths/1000 live births in 
scores of countries, above 30/1000 in a few. And - in those with very high neonatal mortality - infection accounts for a particularly high proportion of all newborn deaths, up to $30 \%$ or more. ${ }^{4}$

From the mid-to-late 1990s - recognizing that the freshly-cut umbilical cord stump is a significant wound and that pathogen exposure of that wound could be a major contributor to newborn infection deaths - programmatic attention was given to improving cord-care at birth and over the following several days, particularly the "cleans" at birth, including use of a clean cutting instrument, a clean tie or clamp, clean hands, and clean surfaces. In many settings, application of various substances to the cord-stump was widespread, some with potentially very high pathogen loads. So, key messaging at that time included keeping the cord stump clean and dry and not applying anything to it. These principles were reflected in recommendations released by WHO in 1998.5 In that guidance, it was acknowledged that inadequate evidence was available on use of antiseptics on the cord stump, and more research was needed.

\section{CHLORHEXIDINE PROGRAM EVOLUTION IN NEPAL}

\section{FROM EVIDENCE FOR EFFICACY TO EXPLORATION OF PROGRAM FEASIBILITY}

From the early 2000s, the Government of Nepal was giving increasing attention to newborn health, as reflected in a National Newborn Health Strategy, adopted in 2004. With this new focus on newborn health and the WHO call for further study on antiseptics for cord care, investigators from the Johns Hopkins Bloomberg School of Public Health began a trial in rural Nepal - in 2002 - testing the effectiveness of chlorhexidine digluconate $7.1 \% \mathrm{w} / \mathrm{v}$ in aqueous solution, applied to the newborn cord-stump, with primary cord infection and newborn death as endpoints. The trial, published in $2006,{ }^{6}$ found chlorhexidine effective in reducing risks of both infection and death. These findings were replicated in subsequent trials (see Box 1).$^{7-10}$

On completion of the trial, results were shared with technical officers in the Ministry of Health $(\mathrm{MoH})$, leaders in the medical community, and others in Nepal. Two USAIDfunded technical agencies (John Snow Inc. - JSI, and the Academy for Educational Development - AED) continued to explore feasibility of moving this intervention towards program use, between 2006-2009, through:

- Continued strategic engagement with a key interested senior Ministry of Health official,

- Formative research on cord-care practices and associated beliefs, including exploration of the properties household-level users would want in a medicinal product for cord-care, ${ }^{11}$

- Engaging with PATH/Seattle to develop product specifications,

- Finding a local pharmaceutical producer open to developing and registering a product and initially providing it in small quantities for research and piloting. This resulted in identifying and cultivating a relationship with a local, family-owned company (Lomus
Pharmaceuticals) that subsequently provided all needed product, ${ }^{12}$

- Conducting a hospital-based, non-inferiority trial that confirmed the effectiveness of a more consumerfriendly gel formulation, ${ }^{13}$

- Conducting a small, quasi-experimental user-preference study, confirming that gel was preferred to liquid. 13

\section{New evidence from beyond Nepal}

During the period 2006-9, similar replication studies were begun in Bangladesh ${ }^{7}$ and Pakistan, ${ }^{8}$ and published in 2011. Both showed marked reductions in risk of omphalitis and newborn death. The Bangladesh study included an arm with only day-of-birth application and found that mortality reduction benefit was at least as great as for multi-day application, although a reduction in omphalitis risk required multi-day application. Two further trials have recently been published, from Zambia ${ }^{9}$ and Tanzania, ${ }^{10}$ also showing reduction in omphalitis risk. Across these 5 trials and others included in a 2015 Cochrane review, ${ }^{14}$ omphalitis risk was reduced by an average of about $50 \%$ and, in settings with high infection-attributable mortality, there was a discernable reduction in mortality risk. In none of the trials were there cases of severe adverse outcomes attributable to the intervention.

\section{THE ROLE OF A WITHIN-GOVERNMENT CHAMPION}

A senior Ministry of Health official with significant influence was identified as a potential champion and engaged in the earliest briefings on results of the trial (before it was published). Over a period of several years, he provided important strategic leadership on chlorhexidine, for example in determining suitable timing for key forward steps and then, through formal and informal processes, ensuring needed government approvals. In 2007, under his leadership, the Ministry convened a national consultative meeting to discuss implications of the findings (see Figure 1). A year later, in 2008, the Ministry formed a Technical Working Group (TWG), which continued - in subsequent years to provide direction for the initiative. In 2009, this official was able to secure approval from the Ministry to move forward with large-scale piloting, implementing through government health services in 4 districts (population about 1.3 million).

\section{REALISTIC PILOTING AT RELATIVELY LARGE SCALE}

Given favorable results from the non-inferiority trial of a gel formulation and evidence that consumers preferred this product, gel was adopted for this pilot. At the time the 


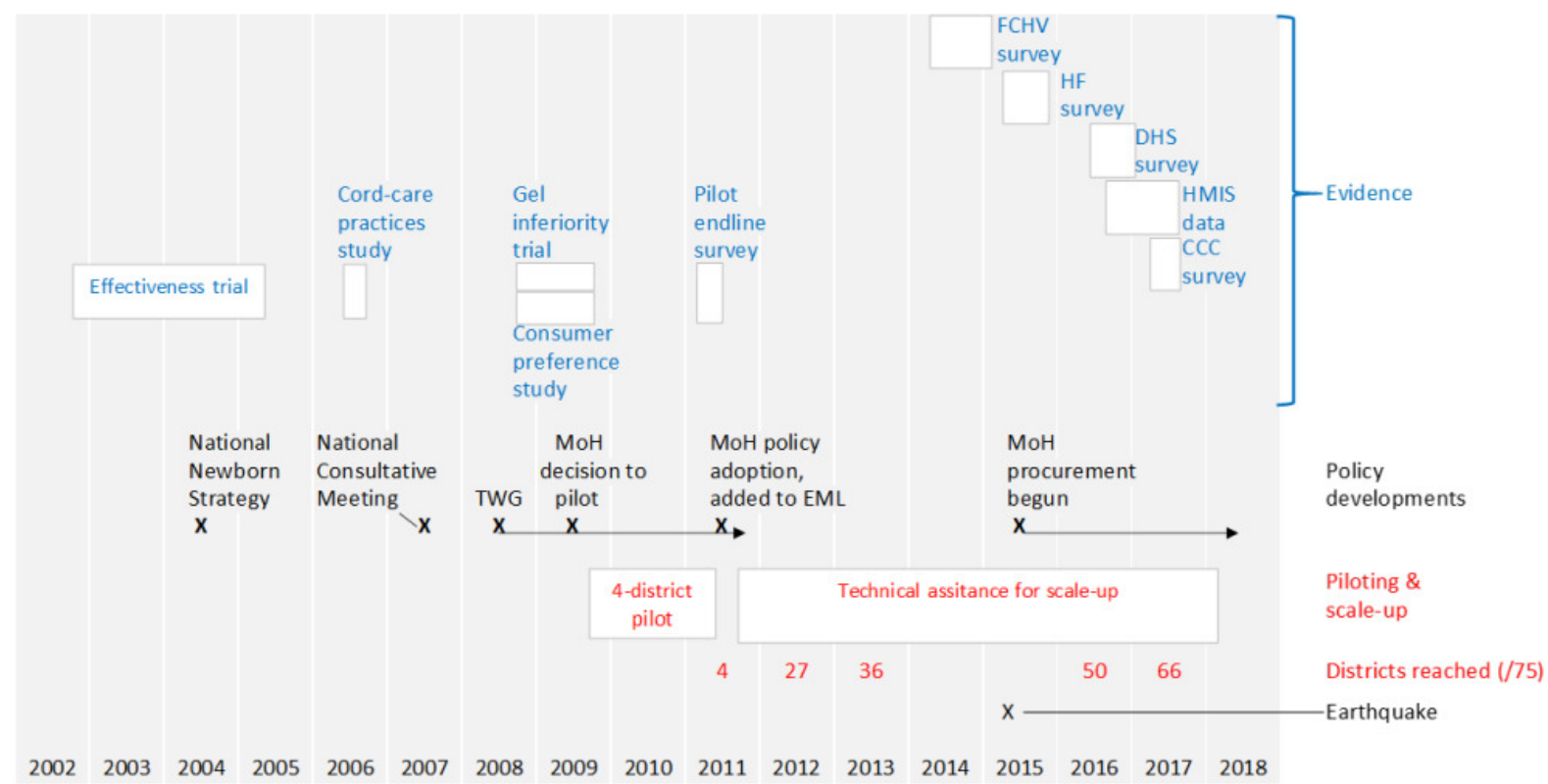

Figure 1. Timeline - evidence, policy and scale-up.

pilot began, $63 \%$ of births were still at home ${ }^{15}$ and, although the pilot implementation strategy included use at facility births, it particularly emphasized advance distribution of chlorhexidine to pregnant women through local female community health volunteers (FCHVs), with the assumption that the mothers themselves or other family members would apply the chlorhexidine. The pilot demonstrated real-world feasibility. A representative household survey of women who had recently given birth ${ }^{16}$ found that $43 \%$ of these women reported having received chlorhexidine during pregnancy. Altogether 57\% reported that chlorhexidine was applied. In the best-performing district, the proportion was $67 \%$; in the poorest-performing, $47 \%$. Comparing observed coverage for births over the first 6 months of implementation to the second 6 months, an improvement was seen, increasing from $53 \%$ to $61 \%$.

\section{POLICY ADOPTION}

With leadership from the Ministry official championing this intervention (including his active involvement in designing an implementation strategy) and drawing on findings of the pilot, in 2011 the government of Nepal formally adopted the new intervention, added chlohexidine to its national Essential Medicines List (EML), and approved a plan for national scale-up with funding support from USAID and technical assistance from JSI, provided through the Chlorhexidine Navi Care Project. The model to be scaled up included:

- Use of a gel product,

- Single application on the day of birth, with no further use on subsequent days (to avoid complicating existing messaging about clean, dry cord-care),
- Use after all facility births as part of early care of the newborn (initially in government hospitals, but later extending to private health facilities),

- Advance distribution through FCHVs to pregnant women in case births happened at home,

- Roll-out strategy piggy-backing introduction of the intervention on other maternal-newborn programs being introduced at that time, notably the Community-Based Newborn Care Package,

- Programmatic integration with other aspects of maternal-newborn health service delivery

It is important that the use of chlorhexidine was understood not as an alternative to but as an integral part of good infection prevention practice around the time of birth.

\section{THE PRODUCT}

The government committed that, from year 2 of the scaleup, it would cover commodity procurement costs. For various reasons (notably, miscommunication with regard to product specifications), there were delays in the government taking this on, but partners were able to cover this expense in the meantime, as the intervention progressively scaled up. The local pharmaceutical company that had registered a gel product and made it available in small quantities for research and piloting, in 2011 went into larger-scale production to support expansion. There were also opportunities from that time for the company to supply in small quantities for pilots in other countries. ${ }^{12}$

\section{SCALE-UP}

As illustrated in Figure 1, chlorhexidine was progressively scaled up across the country over a period of 6 years. Most 
of the leadership and resources required were from the government health system; however, a number of donorfunded partners supported the scale-up effort. As described above, this was done piggy-backing on the roll-out of other maternal-newborn health programs deployed over this period. Across all districts, orientation was given to district program managers, health workers in all government health facilities (and selected private hospitals), and to all FCHVs. The commodity was made available from the time of orientation.

There was, however, some variation in how rollout was done. Depending on which other maternal-newborn activities chlorhexidine introduction was integrated with, time allocated for orientation to health workers and FCHVs on chlorhexidine varied from as little as 3 hours to a full day. In 23 of the 75 districts, after introduction, a dedicated partner-supported staff person remained in the district for at least 1 year, following up the new chlorhexidine program effort, supporting government counterparts to ensure effective institutionalization of the new practice. For the remaining districts, such support was provided on an intermittent basis by visiting project staff. Although there are reasons to believe that the various rollout modalities may have had differential effects on subsequent program performance, the complexity of the situation on the ground and limitations of the available data preclude any firm conclusions.

\section{LEARNING AND ADAPTATION}

In the initial wave of implementation (across 10 districts), the main delivery modality for chlorhexidine was advance distribution by FCHVs to women in the last 2 months of pregnancy, with chlorhexidine also made available for facility births. But results from a mid-term assessment ${ }^{17}$ suggested that this strategy was missing many pregnant women. So, for subsequent rollout, chlorhexidine distribution was also added to routine antenatal care (ANC). The midterm assessment also revealed a lack of awareness of the intervention at population level. Therefore, over the years 2013-2014, program efforts also included mass media promotion of chlorhexidine use for umbilical cord care, using radio and television.

\section{TENSIONS ARISING ON PROGRAM STRATEGY}

During early scale-up, there was no global-level guidance on chlorhexidine for cord care, but from 2013 WHO added chlorhexidine for cord care to its model Essential Medicines List and issued new cord-care guidelines, with support for chlorhexidine use under specified conditions. ${ }^{18}$ The recommendation restricted use to home births since the major trials published to that point had been done in study populations in which most births were at home, although disaggregated analysis of 3,293 facility births from the Nepal and Bangladesh trials showed at least as large effects for omphalitis and mortality risk as for those born at home. ${ }^{19}$ At times, the inconsistencies between the delivery modality pursued by the government and that recommended by WHO created tensions and somewhat undermined momentum, but continuing program effort was sus- tained as key stakeholders and opinion leaders were progressively brought fully on board with the locally adopted strategy.

\section{METHODS}

The study reported here consisted of multiple elements, as outlined in Table 1: a primary study which constituted the main source of data used - the national Chlorhexidine Compliance and Coverage Survey - several other surveys (a national survey of Female Community Health Volunteers, a national Health Facility Survey, the most recent national Demographic and Health Survey), and data from the Ministry of Health's Health Management Information System. We conducted further analysis from each of these sources and are not merely citing published findings.

Our intention, with this analysis was to assess several dimensions of program performance. For this purpose, implementation strength and effective coverage were tracked. By "implementation strength," we mean key measurable determinants required for achieving high effective coverage. ${ }^{20,21}$ In simple terms, 2 key conditions need to be met to achieve high coverage for this intervention: 1) the commodity needs to be reliably available at the point of use, and 2) health workers (or household members) need to be motivated to use it.

We use the term "effective coverage," as defined by Kyei et al. ${ }^{22}$ as: the proportion of a population who need a service that receive it delivered with sufficient quality for it to be effective (Note that earlier, Tanahashi ${ }^{23}$ used the term "effectiveness coverage" to mean essentially the same thing). So, effective coverage entails not just contacts between the health system and its beneficiaries or dispensing a commodity (for example, to pregnant women), but that an efficacious intervention is actually used. In this particular case, we operationalize "effective coverage" as the proportion of newborns who actually had chlorhexidine applied to the cord-stump (see Box 2).

\section{Effective coverage}

A stricter operationalization of "effective coverage” in this case would also include a requirement that chlorhexidine be well-applied to the cut surface of the cord-stump, and that the application happen within the first 24 hours after birth, since studies by Mullany ${ }^{6}$ and Arifeen ${ }^{7}$ provide evidence suggesting that later application may not reduce mortality risk. However, from a practical measurement point of view, such information is unobtainable under usual program conditions.

Figure 2 presents a causal framework for this intervention as implemented in Nepal, showing the relationship between key dimensions of implementation strength and effective coverage (we also used this framework to organize presentation of our findings). 
Table 1. Data sources*

\begin{tabular}{|c|c|c|}
\hline Data source & $\begin{array}{l}\text { Data } \\
\text { collection } \\
\text { period }\end{array}$ & Methods, sample size, data elements, limitations \\
\hline \multirow{3}{*}{ FCHV Survey } & \multirow{3}{*}{$\begin{array}{l}\text { Aug } 2014 \\
- \text { Feb } \\
2015\end{array}$} & $\begin{array}{l}\text { Nationally representative FCHV survey; analysis included } 42 \text { districts in which chlorhexidine had } \\
\text { been introduced. } \mathrm{N}=2,626 \mathrm{FCHVs} \text {. }\end{array}$ \\
\hline & & $\begin{array}{l}\text { This data source was used to assess availability of chlorhexidine with FCHVs, and their reported } \\
\text { frequency of dispensing chlorhexidine. }\end{array}$ \\
\hline & & Dispensing information is based solely on FCHV self-report. \\
\hline \multirow[t]{2}{*}{$\begin{array}{l}\text { Health } \\
\text { Facility } \\
\text { Survey }\end{array}$} & \multirow[t]{2}{*}{$\begin{array}{l}\text { April - } \\
\text { Nov } 2015\end{array}$} & $\begin{array}{l}\text { Nationally representative sample of facilities offering childbirth services. } N=457 \text {. Health } \\
\text { facilities assessed in this survey included all public-sector hospitals, all larger volume private } \\
\text { hospitals, and a representative sample of lower level public facilities. }\end{array}$ \\
\hline & & It was used in our analysis to determine chlorhexidine stock status. \\
\hline \multirow{3}{*}{$\begin{array}{l}\text { Nepal } \\
\text { Demographic } \\
\text { \& Health } \\
\text { Survey } 2016 \\
\text { (NDHS) }\end{array}$} & \multirow{3}{*}{$\begin{array}{l}\text { Jun } 2016 \\
- \text { Jan } \\
2017\end{array}$} & $\begin{array}{l}\text { National multi-stage household cluster survey, representative sample of RDWs (2yr). N=1,978. } \\
\text { Note that, at the time of the survey, chlorhexidine had been introduced in } 56 / 75 \text { districts. }\end{array}$ \\
\hline & & Chlorhexidine applied according to mother (by place of birth) \\
\hline & & For facility births, mothers were not necessarily aware of all procedures done on newborn. \\
\hline \multirow{3}{*}{$\begin{array}{l}\text { Health } \\
\text { Management } \\
\text { Information } \\
\text { System } \\
\text { (HMIS) }\end{array}$} & \multirow{3}{*}{$\begin{array}{l}\text { July } 2016 \\
\text { - July } \\
2017\end{array}$} & $\begin{array}{l}\text { National reporting data, as documented in the Department of Health Services Annual Report } \\
\text { 2073-4. The HMIS is the Ministry's integrated routine health information system that captures } \\
\text { data elements from community level (including FCHV reports), peripheral health facility level, } \\
\text { and hospital level (including private hospitals participating in the government's free maternity } \\
\text { care program). }\end{array}$ \\
\hline & & $\begin{array}{l}\text { Additional data elements for chlorhexidine include: 1) FCHV reports on chlorhexidine use following } \\
\text { home births; and 2) HF reports on chlorhexidine use following institutional births. }\end{array}$ \\
\hline & & $\begin{array}{l}\text { FCHVs only reach a fraction of mothers after birth, so may not accurately report on } \\
\text { chlorhexidine use at home births. }\end{array}$ \\
\hline \multirow{3}{*}{$\begin{array}{l}\text { National } \\
\text { Chlorhexidine } \\
\text { Coverage \& } \\
\text { Compliance } \\
\text { survey }\end{array}$} & \multirow{3}{*}{$\begin{array}{l}\text { April - } \\
\text { Aug } 2017\end{array}$} & $\begin{array}{l}\text { Multi-stage household cluster survey, representative sample of RDWs (1yr) in } 56 \text { program } \\
\text { districts. } N=3,661 \text {. }\end{array}$ \\
\hline & & $\begin{array}{l}\text { Chlorhexidine received during pregnancy, chlorhexidine applied, according to mother's report (by place } \\
\text { of birth) }\end{array}$ \\
\hline & & For facility births, mothers were not necessarily aware of all procedures done on newborn. \\
\hline
\end{tabular}

FCHV - female community health volunteers, RDW - recently-delivered woman

"Data from all of the sources above was analyzed for this paper; ie, we are not merely presenting data that is already published elsewhere.

For this intervention in this setting, an important challenge both for effectively delivering the intervention and for tracking program performance is that during the critical point in the life-course when the intervention needs to be used, women and their newborns can find themselves in quite varied circumstances. Although maternity service utilization is rapidly changing, a significant proportion of births in Nepal still take place at home without the assistance of a suitably skilled and equipped health worker. For such cases, when an intervention requires use of a program commodity, either the commodity needs to be distributed in advance to pregnant women or there needs to be some provision to quickly make it available to women following home births.

Among facility births, one segment of the population gives birth in peripheral-level birthing centers; another gives birth in government hospitals; and another, in private health facilities, many of which are not supplied by the government logistics system and do not routinely report their services into the government's health management information system. For each segment, different programmatic efforts are required to introduce a new intervention and different methods are needed to obtain performance data. Therefore, to track overall population effective coverage a mixed strategy is needed, drawing on multiple data sources.

\section{DATA SOURCES}

This paper presents relevant program performance data from several sources, the primary of which is the Chlorhexidine Coverage and Compliance Survey (CCCS), commissioned by USAID/Nepal and implemented by a local contract research agency (New ERA) over the period May August 2017, capturing information on births between April 2016 and the time of the survey. Of the data sources used for this paper, it was the only one commissioned specifically for assessing performance of the chlorhexidine program. For the other data sources, chlorhexidine-related variables were opportunistically incorporated.

The CCCS provides data on: whether or not women received chlorhexidine during pregnancy (and, if so, from what source), place of birth, and whether or not chlorhexidine was applied (and, if so, by whom). The sampling frame included 56 of Nepal's 75 districts, in which chlorhexidine had been introduced at both hospital level and communitylevel distribution through ANC and FCHVs, as of mid-2016. The survey sample was drawn using multi-stage random sampling, at district, Village Development Committee (roughly equivalent to a rural municipality), and ward levels (with wards as the primary clusters). In each ward, a census listing of all households was done, determining which in- 


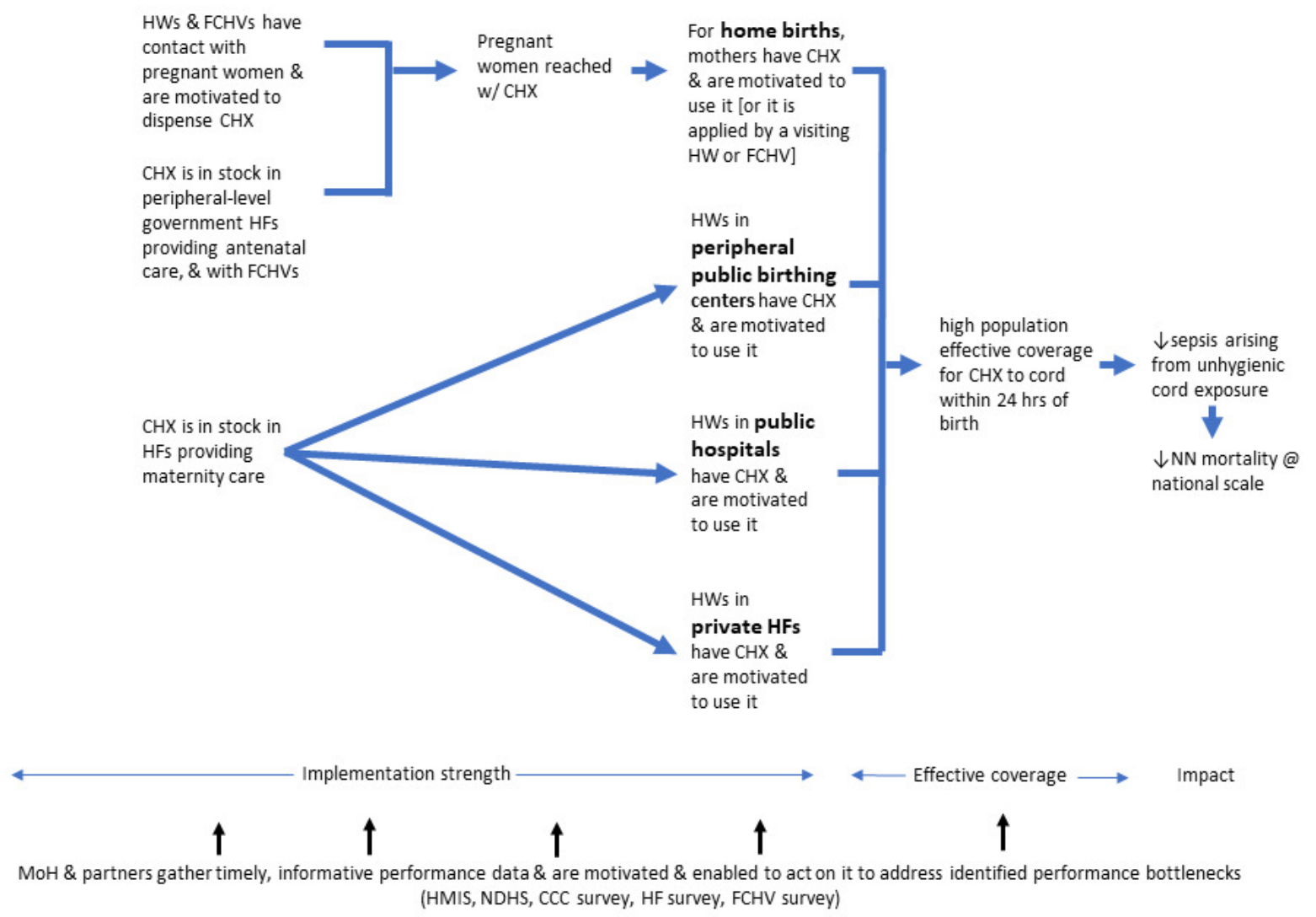

Figure 2. Key steps in the causal process for achieving impact.

HW - health worker, CHX - chlorhexidine, HF - health facility, NN - neonatal, MoH - Ministry of Health

cluded a "recently delivered woman" (a woman reporting a completed pregnancy over the previous year, RDW). In each cluster, 11 RDWs were randomly selected for the full survey questionnaire. A total of 3,661 women were surveyed. Data were analyzed using Stata version 12 (Stata Corp., College Station, Texas). Analysis included disaggregation by wealth quintiles. Ethical approval for the survey was obtained from the Nepal Health Research Council (Ref no. 1785, April 16, 2017).

In addition to the above study, we triangulated findings with other data sources outlined in Table 1.

One reason for use of multiple sources is that any single source gives an incomplete picture (for example, some of these sources are restricted to health facility data). Furthermore, because the various sources have different limitations, by triangulating across more than one we can gain greater insight into what has been happening than from any single source. We have included the government's Health Management Information System not just because it provides an additional perspective, but because it is a routine data source that continues to provide a view on program performance, year by year. Comparing results from HMIS with our survey data source permits cross-validation of findings from this routine data source. This can be useful to program managers.

\section{RESULTS}

As outlined in Figure 2, an adequate assessment of program performance needs to take into account the varied circumstances in which the intervention could be delivered, notably by place of birth. For each such scenario, different program strategies are required to ensure delivery of the intervention and different data sources may be required to track performance. In the analysis below, for each such scenario and associated strategy, we have endeavoured to characterize implementation strength and effective coverage, as discussed earlier.

\section{CHANGING PICTURE WITH REGARD TO PLACE OF BIRTH}

Depending on place of birth, different strategies are required to ensure coverage and different methods are required for measurement. Early on, at the time chlorhexidine was introduced (2009) 63\% of births in Nepal took place at home. ${ }^{15}$ The pattern has rapidly evolved since then. The most recent nationally representative data (NDHS 2016, ${ }^{24}$ restricting to births over the preceding 2 years) show only $36 \%$ of births at home, $12 \%$ in private health facilities, $15 \%$ in peripheral-level government birthing centers, and 33\% in government hospitals. In more recent data from the 56 
districts included in the 2017 CCCS (covering 1 year preceding the survey), home births were even lower - at $27 \%$, with $12 \%$ in private health facilities, $23 \%$ in peripheral-level public facilities, and $37 \%$ in government hospitals.

\section{USE OF CHLORHEXIDINE FOLLOWING HOME BIRTHS}

\section{IMPLEMENTATION STRENGTH}

FCHV and health worker contact with pregnant women: According to CCCS findings, only $55 \%$ of recently delivered women reported having met with their local FCHV during their pregnancy to receive health advice $(\mathrm{N}=3,661)$. From the FCHV survey, among FCHVs in chlorhexidine program districts, 98\% reported having offered health advice to at least 1 pregnant woman over the preceding 3 months. However, clearly they are not reaching all women. One could speculate that those living further away from the FCHV may be less likely to receive such visits. In the CCCS, $92 \%$ of recently delivered women reported having had at least 1 antenatal contact with a suitably trained health worker. The 2016 Nepal DHS survey had similar findings: $94 \%$ of women having given birth over the preceding 5 years reported at least 1 ANC contact. ${ }^{23}$

Chlorhexidine in stock with FCHVs and peripherallevel government health facilities: This is an important requirement to ensure provision to pregnant women, dispensed either by FCHVs or during antenatal contacts. From the FCHV survey (restricting analysis to program districts), through late $2014,52 \%$ of FCHVs $(\mathrm{N}=4,302)$ were found to have chlorhexidine in stock. From the Health Facility Survey, in the 48 districts in which program rollout had been completed by the time of the survey, $78 \%$ of health facilities were found to have chlorhexidine in stock. Chlorhexidine was in stock in $75 \%$ of peripheral-level government facilities offering childbirth services. These were mainly health posts (also offering ANC).

Pregnant women reached with chlorhexidine, by FCHVs and health workers: The national CCCS, conducted through mid-2017, found that in program districts only $20 \%$ of RDWs reported having received chlorhexidine during pregnancy, and $65 \%$ of those from an FCHV, 35\% from antenatal care contact with a health worker. From the $F C H V$ survey, among those based in chlorhexidine program districts, only $29 \%$ of FCHVs reported having either dispensed chlorhexidine to a pregnant woman or applied it to the cord of a newborn at least once over the previous 3 months.

\section{EFFECTIVE COVERAGE FOR HOME BIRTHS}

Use of chlorhexidine, disaggregating by timing of receipt and by person applying: From the national CCCS, one third (33\%) of those having non-institutional births $(\mathrm{N}=988)$ reported that chlorhexidine was applied (fewer than $1 \%$ did not know whether or not it had been applied). Of these, 59\% reported that the chlorhexidine had been obtained during pregnancy, $41 \%$ after the birth. For RDW who obtained chlorhexidine during pregnancy, 55\% reported they applied chlorhexidine themselves or it was applied by another family member. Among RDW obtaining chlorhexi- dine only after birth, $74 \%$ reported it was applied by a FCHV or health worker visiting the home.

From data reported in the government's HMIS for 2016-17, of the expected 641,883 births in the population, there were 377,557 documented in public health facilities plus larger private facilities reporting into HMIS, representing $59 \%$ of total expected births. From both NDHS and national CCCS results, $12 \%$ of births were reported by RDW as having happened in private sector facilities. From available data, it cannot be determined exactly what proportion of these private facility births are captured in HMIS but, if we assume approximately half (ie, $6 \%$ of all births), that would leave $35 \%$ of all births happening in the home, coming to 225,000 . In the government's HMIS data for 2016-17, 95,283 newborns were reported by FCHVs to have had chlorhexidine applied at home. As a proportion of 225,000, that comes to $42 \%$, somewhat higher than the value measured in the national CCCS. However, some fraction of these FCHV-reported cases would have been facility births followed by home application of chlorhexidine; as such, this figure may be compatible with CCCS findings.

From the national CCCS, among women who received chlorhexidine while pregnant and then gave birth at home $(\mathrm{N}=205)$, 92\% reported that chlorhexidine was applied.

\section{USE FOLLOWING HEALTH FACILITY BIRTH}

\section{IMPLEMENTATION STRENGTH}

In stock at health facility providing maternity care, disaggregating by level: From the Health Facility Survey, in districts in which chlorhexidine had been introduced by the time of the survey, chlorhexidine was found to be in stock in $85 \%$ of public hospitals and, as already noted, $75 \%$ of peripheral-level government health facilities offering childbirth care.

\section{EFFECTIVE COVERAGE FOR HEALTH FACILITY BIRTHS}

From the national CCCS: $70 \%$ of women giving birth in a HF reported they know chlorhexidine was applied, 22\% responded that they didn't know whether or not it had been applied, and 6\% reported that it was not. The proportion responding "don't know" was somewhat higher among those giving birth in hospital (25\%) or primary healthcare center (29\%) than those in health posts (16\%), presumably reflecting differences in procedure with regard to periods of separation of the newborn from the mother. If the proportion actually having chlorhexidine applied among those reporting "don't know" was similar to that among those who did know, the total proportion reached among facility births would be $90 \%$.

As discussed for home births, from the data in the government's HMIS for 2016-17, there were 377,557 births documented in public health facilities plus larger private facilities reporting into HMIS, representing 59\% of total expected births. Within this segment, HMIS reports 341,577 newborns having chlorhexidine applied (90\%). This is similar to the proportion of government hospitals found in the Health Facility Survey to have chlorhexidine in stock (85\%). Among an estimated $6 \%$ of all births occurring in private 
Table 2. Coverage among home births, by socio-economic strata

\begin{tabular}{lc|cc}
\hline By level of education & & \multicolumn{2}{|c}{ By wealth quintile } \\
\hline No schooling & $21 \%(17-27)$ & lowest & $27 \%(22-34)$ \\
Primary & $26 \%(19-33)$ & $2^{\text {nd }}$ & $33 \%(27-40)$ \\
Some secondary & $37 \%(31-44)$ & $3^{\text {rd }}$ & $34 \%(26-43)$ \\
School leaving certificate, \& above & $57 \%(48-66)$ & $4^{\text {th }}$ & $38 \%(29-46)$ \\
& & highest & $47 \%(34-59)$ \\
\hline
\end{tabular}

*From CCCS (Chlorhexidine Coverage and Compliance Survey), n=321, \% with 95\% confidence interval).

health facilities not reporting into the HMIS, in light of data on stock availability and reported "routine use" in private Health facilities included in the national Health Facility Survey, it is safe to conclude that chlorhexidine coverage would be substantially lower than $90 \%$.

It is interesting to note that among mothers participating in the CCCS and giving birth in a health facility who reported either that they didn't know whether or not chlorhexidine was applied at the facility or who knew that it wasn't $(\mathrm{N}=588)$, for the majority of their newborns $(55 \%)$ chlorhexidine was subsequently applied at home, either using product obtained during pregnancy or applied by a FCHV or health worker visiting the home.

\section{SOCIO-ECONOMIC AND OTHER FACTORS INFLUENCING COVERAGE}

From national CCCS findings, comparing districts where the program was well-established ( $\geqslant 4$ years since introduction) and those where the program was more recently introduced, no differences were seen in the proportion of pregnant women receiving chlorhexidine and there was little difference in overall coverage for application after birth (data available on request). Among institutional births, there were no differences by wealth quintile in the proportion reporting chlorhexidine application. However, as noted earlier, from NDHS 2016 data, there is a marked disparity for institutional births by wealth quintile, with only $36 \%$ of births among the lowest quintile taking place in a HF vs. $90 \%$ among those in the highest.

Unlike the situation for institutional births, for home births the proportion of women reporting that chlorhexidine was applied was higher among more educated and wealthier women (see Table 2).

\section{PROGRAM COSTS}

By the end of 2017, chlorhexidine had been extended to all districts in the country and was being applied to the cord stumps of approximately $70 \%$ of all newborns on the day of birth. Based on the preventive efficacy demonstrated in the Nepal trial, ${ }^{6}$ use of this intervention in this context can be expected to prevent approximately 1 death per 200 babies protected with chlorhexidine. With approximately 450,000 newborns/ year having chlorhexidine applied (as of 2017), that represents over 2,000 averted deaths per year (although, as we've noted, actual impact is also a function of the vulnerability profile of those reached). At a current unit price for the commodity of US\$ 0.10 , the total annual procurement cost for the government is less than US\$ 50,000 . This is now the most significant recurrent cost associated with delivery of this intervention.

Over the $6 \frac{1}{2}$ year life of the Chlorhexidine Navi Care Program - which provided technical support to government to introduce and roll-out the program - the total cost for this technical assistance (not including commodity procurement) was US $\$ 3.6$ million. The project ended in early 2018.

\section{DISCUSSION}

\section{POPULATION EFFECTIVE COVERAGE}

Although, in principal, it is important for those developing programs to keep their focus on achieving high population effective coverage, as we have noted in this paper even for a simple intervention like chlorhexidine, actual measurement of effective coverage may not be straightforward. From the coverage estimates across these segments, overall coverage was approximately $70 \%$, although this estimate is subject to considerable uncertainty. For births in government health facilities, Nepal has a data element in the national HMIS. It is encouraging that routine reporting shows that chlorhexidine is being used for $90 \%$ or more of facility births. However, such data may not be fully trustworthy; routine health information systems are often beset by over-reporting. Household surveys of recently delivered women are subject to other limitations. As we have noted, the national CCCS found that close to a quarter of women giving birth in health facilities reported being uncertain whether or not chlorhexidine was used. For home births, women were able to give a more certain response.

\section{FACTORS CONTRIBUTING TO RELATIVELY SUCCESSFUL SCALE-UP}

In Nepal, from the first published evidence of intervention effectiveness to nation-wide delivery at comparatively high coverage took 10 years. In global health, that is an unusually short time-frame for such a process. What can account for this better-than-usual experience? The following factors have contributed:

- Robust, locally-generated evidence on effectiveness,

- Government leadership and steady progress towards institutionalization,

- Early and continued substantive engagement between academics who generated the evidence and counterparts in the ministry of health, leaders in the 
medical profession, donors, technical agencies, and the local pharmaceutical industry to maintain and build support,

- Initial piloting under realistic program conditions and ongoing practical evidence generation related to implementation issues, as a basis for directing decisions,

- Deployment of a user-friendly formulation delivered through existing program and service-delivery platforms,

- Rigor in tracking program performance, using routine monitoring and special studies to characterize actual coverage, taking action to address performance gaps and - where necessary - revising delivery strategy

\section{CHLORHEXIDINE PRODUCT AVAILABILITY}

As discussed in the Introduction, from 2011 a local drug company, Lomus, began to engage, developing a suitable product, registering it with the national drug regulatory agency, and later producing it in sufficient volume to meet national requirements. ${ }^{12}$ Earlier in the rollout, donor-supported partner agencies were purchasing needed supplies. From 2015, the government has been procuring chlorhexidine and distributing it through its district public health offices (the first year, procurement was done under special arrangements related to the earthquake response; regular procurement began the following year). Private health facilities participating in the government's free maternity care program have generally been obtaining their supply through local district public health offices. Some non-participating private health facilities are purchasing from Lomus or its local suppliers. So there has been some degree of institutionalization of procurement and supply chain management. As noted earlier, as of mid-2015 most public health facilities were found to have chlorhexidine in stock. However, only half of FCHVs had any chlorhexidine. So, clearly, one important barrier to making chlorhexidine available during pregnancy, particularly for women likely to give birth at home, is weaker supply chain provision to the FCHV level.

An additional challenge, moving forward, is that there have recently been major politico-administrative changes in Nepal, with functions devolved from national government to newly-formed provincial governments and municipalities, including responsibility for procurement of most health commodities. As with many other health commodities, there are new uncertainties about reliable procurement and distribution of chlorhexidine to point of use.

\section{REACHING THE MOST VULNERABLE: NEWBORNS DELIVERED AT HOME}

At the time chlorhexidine for cord-care was first piloted, most births were at home and the main implementation strategy was advance distribution of chlorhexidine by FCHVs during contacts with women late in pregnancy. During piloting and early scale-up, monitoring data suggested that this strategy was missing many women. As a result, advance distribution was extended to include antenatal contacts. However, this strategy is still failing to reach the majority of newborns delivered at home. The commodity supply chain is not reliably delivering the commodity to the FCHV level and even when it is in stock in peripheral health facilities or with FCHVs, it is not consistently being dispensed to pregnant women. The reasons for this are unclear. Although a high proportion of women have at least some ANC, and over half of women report some contact with their local FCHV during pregnancy, we do not have data on what proportion of women have either an ANC visit or a contact with their FCHV during the final 2 months of pregnancy, the time when chlorhexidine is to be dispensed. Furthermore, there is some anecdotal evidence suggesting that for health workers and FCHVs encouraging women to give birth in health facilities, they may feel conflicted if they are also dispensing products only to be used in case the women delivers at home.

Despite the advance distribution strategy not performing well, it appears that mothers, FCHVs and health workers are aware of the value of chlorhexidine and do make efforts to ensure that, for home births (and indeed following facility births if they're not certain chlorhexidine was applied), chlorhexidine is obtained and applied. It is encouraging that, in this instance, survey findings and HMIS reporting produce similar estimates.

Although facility births have increased over the past decade, most women in the lowest wealth quintiles still give birth at home and are less likely to have applied chlorhexidine. These newborns are at higher risk of life-threatening infection and would particularly benefit from an intervention like chlorhexidine for cord care.

Earlier, it was explained that, as a measure of overall program performance, we are interested in "effective coverage" which Kyei et al. ${ }^{22}$ define as: the proportion of the population who need a service that receive it with sufficient quality (for it) to be effective. This is a simpler (and arguably more practical) definition for "effective coverage" than that used by Shengelia and colleagues ${ }^{25}$ who first introduced the term; they also included a "need" component, the idea being that depending on need, the benefit accruing from uptake of an intervention varies. Not taking need into account, we can have a situation where most of those captured in the numerator of an effective coverage measure may be relatively low need.

Newborns of poorer, less well-educated mothers are, on average more likely to be of low birthweight and to be exposed to unhygienic conditions and, therefore, more likely to benefit from chlorhexidine use. So, using simple measures of population coverage that are not adjusted for vulnerability profile may overestimate expected impact. So, although - based on our available data - overall population coverage for this intervention was about $70 \%$, because those at highest risk are considerably more likely to be born at home and less likely to receive the intervention, the actual population-level impact of this intervention is likely to be considerably less than $70 \%$ of its potential impact.

\section{LIMITATIONS}

As has been acknowledged earlier, each of the data sources used has associated limitations. This is part of the rationale for drawing on multiple sources, allowing for triangulation and cross-validation. The major data source for this paper 
- the Chlorhexidine Coverage and Compliance Survey - was used to determine what proportion of recently delivered women were given chlorhexidine during pregnancy, and what proportion of newborns had chlorhexidine applied to the cord. As with any such household survey, participant recall can compromise accuracy. Specifically, in this case, it was evident that a significant minority of women giving birth in health facilities were unable to say whether or not chlorhexidine had been applied. In the FCHV survey, stock status for program commodities was checked directly; FCHVs were also asked to report on their activities over the previous 3 months. There was no way to cross-validate these self-reports; they could therefore be affected by social acceptability bias. In any case, this source does not give a direct measure of population coverage for chlorhexidine. The Health Facility Survey included a representative sample of government health facilities where antenatal and childbirth care were offered, and provided an opportunity to directly determine stock status, at the time of the survey. However, this dimension of program performance certainly changes over time, and stock status at the time of the survey may or may not be representative of patterns over time. The Nepal Demographic and Health Survey provided another measure of mother's report on whether or not chlorhexidine was used, and is subject to the same data limitations as the CCCS. The Ministry's Health Management Information System is certainly affected by the usual issues of data completeness and accuracy of such systems. However, it is encouraging that findings from this source were broadly in line with those of the surveys.

\section{CONCLUSIONS}

Chlorhexidine for cord-care is a simple intervention but even simple interventions do not deliver themselves, at scale, at high coverage. This paper has described the evolution of a program effort seeking to introduce an efficacious intervention. The government and partners have made serious efforts to achieve and sustain high coverage.

If we look at this as a "glass half-full" story, this effort is now reaching approximately 2 out of every 3 newborns in the country and likely preventing about 2,000 deaths per year at a cost of about US\$ 400/averted death. This is a notable accomplishment.

However, if we look at this as a "glass half-empty" story, we see that the one third of newborns not reached are disproportionately those born among the poorest, most disadvantaged families, and therefore at the highest risk of the early infection-related deaths that this intervention is intended to prevent. The primary strategy to try to reach this segment of the population has been advance distribution to pregnant women, either through ANC or FCHV contacts. But this strategy stands in tension with serious efforts in Nepal to encourage women to give birth in a health facility. The Ministry of Health and its partners need to strengthen their efforts to ensure that front-line service providers are taking advantage of all pregnancy contacts to dispense important interventions for use at birth in case the woman ends up delivering at home; this includes chlorhexidine for cord-care, as well as misoprostol for preventing post-partum hemorrhage, ${ }^{26}$ and counseling on key prac- tices at childbirth and over the first hours and days that follow.

Achievement of population coverage of approximately $70 \%$ has been facilitated by: central procurement and distribution of the commodity by the national Ministry of Health; and focused attention from a dedicated technical assistance project (see Box 3). With devolution and decentralization of procurement and closing of the technical assistance project, there will be new challenges to sustain the gains that have been made. If Nepal is to continue to prevent about 2,000 infection-related newborn deaths per year, with this intervention, and to make further headway among the higherrisk segment giving birth at home, the national Ministry of Health, provincial Ministries of Social Development, and their partners will certainly need to give serious attention to reaching the most disadvantaged and to ensuring procurement and distribution of key program commodities like chlorhexidine.

\section{Key lessons}

Plans generally need revision, if we're committed to good program performance. Conditions present at the time of original design often change over time and assumptions made sometimes turn out not to be warranted (e.g. service providers or community members may not behave as we expect them to).

It can be challenging figuring out what's actually happening - what's important isn't always readily measurable and what can be easily measured isn't necessarily a good proxy for what we are most interested in. It can be helpful to triangulate, making use of multiple data sources, each of which - on its own - would give an inadequate picture.

Sustained serious attention by managers and service providers is a requirement for good program performance (flexible fidelity)

Even when introducing single interventions, one can achieve synergies and greater efficiency by integrating rollout with other related efforts and incorporating performance measurement in related surveys and monitoring systems.

\section{ACKNOWLEDGEMENTS}

The authors acknowledge the important contributions to the success of this program made by health workers and managers in the Government of Nepal Health Services and to the staff of the now completed Chlorhexidine Navi Care Program. Appreciation is also expressed to Save the Children, Saving Newborn Lives, for support provided, including related to documenting implementation strength and effective coverage. 


\section{ETHICS APPROVAL AND CONSENT TO PARTICIPATE}

For the main dataset for this article, the Chlorhexidine Coverage and Compliance Survey, the study was reviewed and approved by the Institutional Review Board of the Nepal Health Research Council.

\section{CONSENT FOR PUBLICATION}

All authors have provided consent for publication.

\section{AVAILABILITY OF DATA AND MATERIAL}

Data from the Chlorhexidine Coverage and Compliance Survey will be available on the USAID Data Development Library, https://www.usaid.gov/data. Data from the Nepal Demographic and Health Survey and from the National Health Facility Survey are available on the http://dhsprogram.com/ website. Data from the Government of Nepal Health Management Information System are available in aggregate form on the http://dohs.gov.np/ website.

\section{FUNDING}

This study was conducted with generous support of the American people through the United States Agency for International Development; through 2 projects: 1 ) the Nepal
Monitoring, Evaluation, and Learning (MEL) Project (CAMRIS International), Evaluation Mechanism Number: AID-367-C-15-00001; 2) and the Chlorhexidine Navi Care Program (JSI Research and Training Institute), AID-OAAA-11-00073. The views expressed in this article are solely the views of the authors and do not reflect the views of the United States Agency for International Development or the United States Government.

\section{COMPETING INTERESTS}

The authors completed the Unified Competing Interest form at http://www.icmje.org/coi disclosure.pdf (available upon request from the corresponding author), and declare no conflicts of interest/

\section{CORRESPONDENCE TO:}

Stephen Hodgins, MD MSc DrPH

University of Alberta | School of Public Health | Edmonton Clinic Health Academy

11405 - 87 Ave | Edmonton AB | Canada T6G 1C9

shodgins@ualberta.ca 


\section{REFERENCES}

1. Lawn JE, Kerber K, Enweronu-Laryea C, Cousens S. 3.6 million neonatal deaths - what is progressing and what is not? Semin Perinatol. 2010;34(6):371-386. do $\mathrm{i}: 10.1053 / \mathrm{j}$. semperi.2010.09.011

2. World Health Organization. Maternal and Neonatal Tetanus (MNT) elimination. Accessed July 16, 2018. $\underline{\mathrm{h}}$ ttp://www.who.int/immunization/diseases/MNTE_init iative/en/

3. Liu L, Johnson HL, Cousens S, et al. Global, regional, and national causes of child mortality: an updated systematic analysis for 2010 with time trends since 2000. Lancet. 2012;379(9832):2151-2161. doi:1 $\underline{0.1016 / \mathrm{s} 0140-6736(12) 60560-1}$

4. Baqui AH, Williams E, El-Arifeen S, et al. Effect of community-based newborn care on cause-specific neonatal mortality in Sylhet district, Bangladesh: findings of a cluster-randomized controlled trial. J Perinatol. 2015;36(1):71-76. doi:10.1038/ip.2015.139

5. World Health Organization. Care of the Umbilical Cord: A Review of the Evidence. WHO; 1998. Accessed July 16, 2018. http://bvsper.paho.org/videosdigitales/ matedu/ICATT-AIEPI/Data/Care\%20of\%20the\%20um bilical\%20cord/fd38a9b4-bfa1-4735-9a9a-e7dbb0faa7 20.pdf

6. Mullany LC, Darmstadt GL, Khatry SK, et al. Topical applications of chlorhexidine to the umbilical cord for prevention of omphalitis and neonatal mortality in southern Nepal: a community-based, clusterrandomised trial. Lancet. 2006;367(9514):910-918. do i:10.1016/s0140-6736(06)68381-5

7. Arifeen SE, Mullany LC, Shah R, et al. The effect of cord cleansing with chlorhexidine on neonatal mortality in rural Bangladesh: a community-based, cluster-randomised trial. Lancet.

2012;379(9820):1022-1028. doi:10.1016/s0140-6736(1 1) $61848-5$

8. Soofi S, Cousens S, Imdad A, Bhutto N, Ali N, Bhutta ZA. Topical application of chlorhexidine to neonatal umbilical cords for prevention of omphalitis and neonatal mortality in a rural district of Pakistan: a community-based, cluster-randomised trial. Lancet. 2012;379(9820):1029-1036. doi:10.1016/s0140-6736(1 1)61877-1

9. Semrau KEA, Herlihy J, Grogan C, et al. Effectiveness of $4 \%$ chlorhexidine umbilical cord care on neonatal mortality in Southern Province, Zambia (ZamCAT): a cluster-randomised controlled trial. Lancet Glob Health. 2016;4(11):e827-e836. doi:10.101 6/s2214-109x(16)30215-7
10. Sazawal S, Dhingra U, Ali SM, et al. Efficacy of chlorhexidine application to umbilical cord on neonatal mortality in Pemba, Tanzania: a community-based randomised controlled trial. Lancet Glob Health. 2016;4(11):e837-e844. doi:10.1016/s221 4-109x(16)30223-6

11. Tuladhar S, Ban B. A Study on Cord Care Practices in Bardiya District. Nepal Family Health Program; 2007. Accessed July 16, 2018. https://www.healthynew bornnetwork.org/hnn-content/uploads/Tuladhar-et-a 1_Bardiya-district_Nepal_NFHP_2007.pdf

12. Oyloe P, Khanal L, Hodgins S, Pradhan ST, Dawson $\mathrm{P}$. Innovative product development partnership reduced neonatal mortality in Nepal through improved umbilical cord care. Health Aff (Millwood). 2017;36(11):1973-1978. doi:10.1377/hlthaff.2017.054 $\underline{8}$

13. Hodgins S, Thapa K, Khanal L, et al. Chlorhexidine gel versus aqueous for preventive use on umbilical stump: a randomized noninferiority trial. Pediatr Infect Dis J. 2010;29:999-1003. doi:10.1097/inf.0b013e 3181e70c59

14. Sinha A, Sazawal S, Pradhan A, Ramji S, Opiyo N. Chlorhexidine skin or cord care for prevention of mortality and infections in neonates. Cochrane Database Syst Rev. 2015;3:CD007835. doi:10.1002/146 51858.cd007835.pub2

15. Ministry of Health and Population [Nepal], New ERA, Macro International Inc. Nepal Demographic and Health Survey 2011. Ministry of Health and Population, New ERA, and ICF Macro International Inc.; 2012.

16. Nepal Family Health Program. Coverage and Compliance of Chlorhexidine in Banke, Jumla and Bajhang Districts. NFHP II; 2012. Accessed July 16, 2018. http://nfhp.jsi.com/Res/Docs/CoverageandCom plianceofKawachinBankeJumlaandBajhanjdistricts201 1.pdf

17. Basnet J. Mid-Term Assessment on Use of Chlorhexidine for Better Cord Care. NewERA; 2013.

18. World Health Organization. WHO Recommendations on Postnatal Care of the Mother and Newborn. Accessed August 13, 2018. http://apps.who.i nt/iris/bitstream/handle/10665/97603/978924150664 9 eng.pdf;jsessionid=17EA0C78BB537BEE93300CEF5 360AE7E?sequence $=1$ 
19. Imdad A, Mullany LC, Baqui AH, et al. The effect of umbilical cord cleansing with chlorhexidine on omphalitis and neonatal mortality in community settings in developing countries: a meta-analysis. BMC Public Health. 2013;13(Suppl 3):S15. doi:10.118 6/1471-2458-13-s3-s15

20. Bryce J, Requejo JH, Moulton LH, Ram M, Black RE. A common evaluation framework for the African Health Initiative. BMC Health Serv Res.

2013;13(S2):S10. doi:10.1186/1472-6963-13-s2-s10

21. Diaz T, Guenther T, Oliphant NP, Muñiz M. iCCM Symposium impact outcome evaluation thematic group. A proposed model to conduct process and outcome evaluations and implementation research of child health programs in Africa using integrated community case management as an example. J Glob Health. 2014;4(020409).

22. Tanahashi T. Health service coverage and its evaluation. Bull World Health Organ. 1978;56:295-303.
23. Kyei NNA, Chansa C, Gabrysch S. Quality of antenatal care in Zambia: a national assessment. BMC Pregnancy Childbirth. 2012;12(1):151. doi:10.118 6/1471-2393-12-151

24. Ministry of Health and Population [Nepal], New ERA, Macro International Inc. Nepal Demographic and Health Survey 2016. Ministry of Health and Population, New ERA, and ICF Macro International Inc.; 2017.

25. Shengelia B, Tandon A, Adams OB, Murray CJL. Access, utilization, quality, and effective coverage: an integrated conceptual framework and measurement strategy. Soc Sci Med. 2005;61(1):97-109. doi:10.1016/ j.socscimed.2004.11.055

26. Rajbhandari SP, Aryal K, Sheldon WR, et al. Postpartum hemorrhage prevention in Nepal: a program assessment. BMC Pregnancy Childbirth. 2017;17(1):169. doi:10.1186/s12884-017-1347-z 